\title{
EFFECT OF ACETYL-L-CARNITINE ON LEUKEMIA L1210 RESISTANT TO MITOXANTRONE
}

\author{
Mohamed Niang, Milan Mělka \\ Charles University in Prague, Faculty of Medicine in Hradec Králové: Department of Medical Biochemistry
}

\begin{abstract}
Summary: Supportive care in tumour chemotherapy is a subject of intensive research. The complications of cytostatic therapy are a cause of extensive research of their pharmacological interactions and side effects. The immunologic and biochemical changes accompanying tumours are the factor that is most responsible for the worsening of the physiology of the host. Regimens containing carnitine and it's acetyl-derivative are used in many cases, among others even for preventing hepatotoxicity. Our hypothesis was to verify the supporting metabolic effects of acetyl-L-carnitine hydrochloride (ALC) in combined therapy with mitoxantrone (MX) and hepatotoxic cytostatic drugs including alkylating agents. This present report describes the effect of ALC in combination with MX on DBA/2 male mice bearing a transplantable L1210 leukemia resistant to MX. The criterion for evaluation of effect was the length of survival time of experimental animals. The proportional-hazards model quadratic in the drug dose (7) was used for survival time evaluation and optimal dose calculation. The hazard functions and the index of relative hazard were determined using Weibull distribution after logarithmic transformation of the entered data in each particular group. The dose-response curve was represented by a second-degree polynomial without absolute term. The combination therapy revealed that the optimal dose of ALC was $186 \mathrm{mg} / \mathrm{kg}$ s.c. This relation is shown in Fig.1. A significant effect of ALC (s.c.) in combined therapy with MX (6 mg/kg i.v.) given to animals bearing an experimental form of leukemia L1210/MX resistant to MX was proven at a level of probability $\mathrm{p} \leq 0.001$. The effect of ALC in monotherapy was not demonstrable.
\end{abstract}

Key words: Mitoxantrone dihydrochloride (MX); Acetyl-L-carnitine hydrochloride (ALC); Protective effect and L1210 leukemia

\section{Introduction}

Mitoxantrone dihydrochloride, a synthetic anthraquinone, is a potent antineoplastic agent and active substance of REFADOR Inj. PLIVA-LACHEMA. The chemical structure and chemical name are:<smiles>O=C1c2c(O)ccc(O)c2C(=O)c2c(NCCNCCO)ccc(NCCNCCO)c21</smiles>

1,4-dihydroxy-5,8-bis [2-[(2-hydroxyetyl)amino]ethylamino] anthracene-9,10-dione dihydrochloride.

This active component of the preparation is manufactured by the Research Institute of Organic Synthesis (VÚOS) (17) Pardubice, Czech Republic.
Mitoxantrone (MX) can be used alone and in combination with other agents against various types of neoplasias, including solid tumours (8) and haematological malignancies $(6,21)$. Toxic effects of anticancer therapeutics and acquired cell resistance to these agents, occurring in the course of therapy, are the limiting factors of successful cancer treatment $(14,20)$.

Among substances used to give metabolic support, were trying pre-clinically to determine whether some L-carnitine derivatives, in combination with MX could ameliorate host's metabolic response to tumour processes. The aim was to document new possibilities of using a combination of chemotherapeutics with substances that modulate their therapeutic and toxicologic profiles and that could be of clinical importance as new antitumour drugs and new therapeutic protocols.

In this work we investigated the therapeutic benefit of acetyl-L-carnitine (ALC) in combination with MX on a murine leukemia L1210 resistant to MX.

\section{Materials and methods}

Mitoxantrone (batch No 12/309 VÚOS) was purchased from the Research Institute of Pharmacy and Biochemistry 
in Prague. It is a dark blue hygroscopic crystalline substance, soluble in water, physiological saline and isotonic dextrose solution, with a molecular weight of 517,41.

Acetyl-L-carnitine hydrochloride, a white hygroscopic crystalline substance, with a molecular weight of 239,70 with CAS registry No 5080-50-2. It was a kind gift from Lonza LTD Organic Chemicals Basle, Switzerland.

The antineoplastic activity of MX combined with ALC, was evaluated in vivo on a transplantable L1210 leukemia variant selected for resistance to MX (L1210/MX).

\section{Methods}

DBA/2 male mice from Velaz a.s. weighing 21,3-24,8 $\mathrm{g}$ were used. L1210 cell suspension was intraperitoneally inoculated $\left(2.10^{5}\right.$ cells from the ascites fluid in $0.2 \mathrm{ml}$ of physiological saline per mouse). $80 \mathrm{DBA} / 2$ mice bearing this tumour transplant were divided into 8 groups, a control group and 7 test groups of 10 animals. Animals in the test groups were treated with intravenous (i.v.) or intraperitoneal (i.p.) administrations of MX in a single dose of $6 \mathrm{mg} / \mathrm{kg}$ combined with different doses of ALC ranging from 50 $200 \mathrm{mg} / \mathrm{kg}$. ALC was administered either subcutaneously (s.c.) or intraperitoneally (i.p.).

The proportional-hazards model quadratic (a) in the drug dose (7) was used for evaluation of the survival time and optimal dose calculation.

$$
\lambda_{(\mathrm{t})}=\lambda_{0(\mathrm{t})} \exp \left(\beta_{1} \mathrm{x}+\beta_{11} \mathrm{x}^{2}\right)
$$

where $\lambda_{(\mathrm{t})}$ and $\lambda_{0(\mathrm{t})}$ are the hazard functions at time $\mathrm{t}, \mathrm{x}$ is the dose of ALC in $\mathrm{mg} / \mathrm{kg}$ combined with a single dose of MX, $B_{1}$ and $\beta_{11}$ are coefficients of the second-degree polynomial (a) without absolute term. Hazard functions and the index of relative hazard $\mathrm{R}(\mathrm{b})$

$$
\mathrm{R}=\lambda_{(\mathrm{t})} / \lambda_{0(\mathrm{t})}
$$

were calculated using parameters of Weibull distribution after logarithmic transformation of entered data of each particular group. The dose-response curve was represented by a second-degree polynomial without absolute term.

\section{Results}

Antineoplastic activity of MX combined with ALC was evaluated on transplantable mouse L1210 leukemia in vivo. The combination therapy has been investigated on a murine model DBA/2/L1210 leukemia selected for resistance to mitoxantrone. MX was administered at a single dose of $6 \mathrm{mg} / \mathrm{kg}$.i.v. or i.p. ALC was given in doses ranging from $50-200 \mathrm{mg} / \mathrm{kg}$ (Tab.1). Survival of the experimental animals was observed for 90 days following leukemia inoculation.

For all tested groups, the statistical evaluation of the functional dependence of survival on doses of MX i.v. alone or in combination with ALC s.c. revealed that the F-value (Fisher-Snedecor F-test used for evaluation of the statistical significance of the model) 9.765 was greater than the critical value for the combination of these two drugs at 0.001 level of probability (Tab.2). Thus ALC (s.c.) in combined therapy improved the therapeutic effect of MX dose dependently and significantly. The dose-response function of s.c-administered ALC in combination with MX $6 \mathrm{mg} / \mathrm{kg}$ i.v. appears in Fig.1. The optimal dose of ALC in combination with MX was $186 \mathrm{mg} / \mathrm{kg}$ s.c.

Tab. 1: Antitumour activity of mitoxantrone dihydrochloride (i.v. or i.p.) and acetyl-L-carnitine hydrochloride (s.c. or i.p.) on L1210/MX leukemia bearing DBA/2 mice.

In vivo effect of combination therapies of MX $6 \mathrm{mg} / \mathrm{kg}$ (i.v.) or (i.p.) and ALC at doses of 200, 100 and $50 \mathrm{mg} / \mathrm{kg}$ (s.c.) or (i.p.) on DBA/2 mice bearing a transplantable leukemia L1210 variant resistant to mitoxantrone. Treatment $1 \mathrm{x} 1$ began the first day following the intraperitoneal inoculation of the tumour on day 0 . The table shows the survival days and compares the effect of MX administered (i.v.) versus (i.p.).

\begin{tabular}{|l|c|c|rrrrrrrrrr|}
\hline Group & $\mathrm{N}$ & Dose & \multicolumn{10}{|c|}{ Survival days } \\
\hline Control & 10 & - & 5 & 6 & 15 & 15 & 18 & 19 & 19 & 19 & 19 & 19 \\
\hline MX & 10 & 6 i.v & 4 & 6 & 9 & 9 & 12 & 12 & 13 & 13 & $>90$ & $>90$ \\
ALC & & 0 & & & & & & & & & & \\
\hline MX & 10 & 0 & 10 & 13 & 14 & 15 & 16 & 17 & 18 & 18 & 20 & $>90$ \\
ALC & & 200 s.c. & & & & & & & & & & \\
\hline MX & 10 & 6 i.v. & 14 & 16 & 17 & 17 & 17 & 17 & 17 & 17 & 18 & 18 \\
ALC & & 200 s.c. & & & & & & & & & & \\
\hline MX & 10 & 6 i.v. & 7 & 9 & 11 & 11 & 12 & 16 & 17 & 17 & 18 & 18 \\
ALC & & 100 s.c. & & & & & & & & & & \\
\hline MX & 10 & 6 i.v. & 6 & 7 & 13 & 15 & 16 & 16 & 17 & 17 & 18 & 18 \\
ALC & & 50 s.c. & & & & & & & & & & \\
\hline MX & 10 & 6 i.p. & 18 & 26 & 26 & 27 & 34 & 37 & 37 & 38 & 39 & 60 \\
ALC & & 0 & & & & & & & & & & \\
\hline MX & 10 & 6 i.p. & 6 & 26 & 33 & 33 & 33 & 35 & 35 & 36 & 39 & $>90$ \\
ALC & & 100 i.p. & & & & & & & & & & \\
\hline
\end{tabular}

Tab. 2: Effect of acetyl-L-carnitine hydrochloride (ALC) in combination with mitoxantrone on L1210 leukemia bearing $\mathrm{DBA} / 2$ mice.

Parameter estimates of the proportional-hazards model quadratic in the drug dose, statistical evaluation of the second-degree polynomial and optimal dose calculation.

\begin{tabular}{|l|c|c|c|c|c|c|}
\hline Parameter & SD & $\begin{array}{c}\text { Student‘s } \\
\text { t-value }\end{array}$ & $\mathrm{N}$ & $\mathrm{SD}$ & $\mathrm{r}$ & $\mathrm{F}$ \\
\hline$\beta_{1}=-0.021147$ & 0,004492 & 4,707 & 38 & 0,8642 & 0,5985 & 9,765 \\
\hline$\beta_{11}=0.569359 .10^{-4}$ & $0,2492.10^{-4}$ & 2,285 & & & & \\
\hline
\end{tabular}

Status: ALC active $(\mathrm{p} \leq 0.001)$. Optimal dose $186 \mathrm{mg} / \mathrm{kg}$ s.c.

$\beta_{i}$ - estimated value of the parameter

$\mathrm{N}$ - number of evaluated subjects

SD - standard deviation

$\mathrm{r}$ - correlation coefficient

$\mathrm{F}$ - Fisher-Snedecor test value

$\mathrm{F}=\mathrm{r}^{2}(\mathrm{~N}-\mathrm{p}-1) /\left(1-\mathrm{r}^{2}\right) \mathrm{p}, \mathrm{p}$ - number of parameters. 
Dose-response curve for acetyl-L-carnitine hydrochloride (ALC).

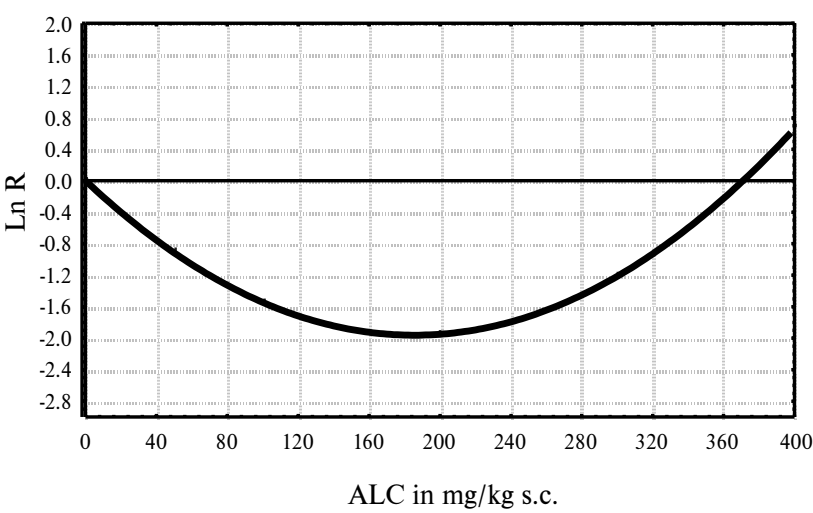

Fig. 1: Dose-response curve for acetyl-L-carnitine hydrochloride (ALC) in the study of survival of DBA/2 mice bearing leukemia L1210/MX resistant to mitoxantrone. The figure shows the dependence of the index of relative hazard R on doses of ALC. The optimal dose of ALC in combination with MX (6mg/kg i.v.) was found to be $186 \mathrm{mg} / \mathrm{kg}$ s.c.

The length of survival of experimental animals bearing a variant form of leukemia resistant to $\mathrm{MX}$ was not statistically different at the dose of MX $6 \mathrm{mg} / \mathrm{kg}$ i.v. compared to control group (non-parametric test, $\mathrm{p} \leq 0.05$ ). In combined therapy with MX (i.p.), ALC at dose of $100 . \mathrm{mg} / \mathrm{kg}$ (i.p.) did not improve the therapeutic effect of MX (non-parametric test, $\mathrm{p} \leq 0.05$ ).

The experiment demonstrated a statistically significant effect of ALC (s.c.) when combined with MX i.v., whereas the effect of ALC (s.c.) alone was not proven.

\section{Discussion}

For most chemotherapeutic regimens, there is a relationship between the dose of drug given and the likelihood of tumour shrinkage (i.e. higher doses give greater tumour cell kill). However, as drug doses are increased, toxicity increases. As opposed to many other classes of drugs, the dose of an antineoplastic agent adequate to achieve tumour cell kill often causes toxicity to normal tissues (15).

The primary toxicities of MX are similar to those seen with the anthracyclines: myelosuppression, nausea, vomiting and cardiac toxicity. Cancer cells are very effective in developing biochemical mechanisms that allow for cellular resistance to a particular antineoplastic agent. In order to overcome the problem of resistance development, more than one drug is generally used to treat a cancer (combination chemotherapy). For instance, most of the topoisomerase II targeted drugs develop cellular resistance through either a mutation in topoisomerase II, decreased topoisomerase II production or through production of $p$-glycoprotein conferring multi-drug resistance $(3,10)$. Drugs used in combination therapies generally have different cellular targets to avoid development of drug resistant tumour cell lines.

Four potentially significant effects of anthracyclines and MX have been described. First, the planar moiety can insert between base pairs of DNA (intercalation). This intercalation was originally felt to be the mechanism for anthracycline cytotoxity. Second, these agents inhibit topoisomerase II. It was not until 1984 that the anthracyclines were found to inhibit topoisomerase II (25). This appears to be the primary mechanism for tumour cytotoxicity. Third, the anthracyclines modify the ability of nuclear helicases to dissociate duplex DNA into single DNA strands, thus hindering the process of strand separation (2). Finally, all clinically active anthracyclines are anthraquinones. As true of all quinones, the anthracyclines can undergo one and two electron reduction producing reactive compounds which damage macromolecules and lipid membranes (19). It is now known that reduced metals (such as iron) are critical components in the formation of these reactive intermediates (5). Free-radical formation induced by the anthracyclines appears to be the primary mechanism by which this class of drugs causes damage to heart tissue. With commonly used dosages, approximately twice as much mitoxantrone can be given before heart failure develops when compared to doxorubicin (18).

The degree of severity of toxic effect is a real challenge to successful treatment. Toxicity modulating agents are now clinically used.

Myelotoxicity is clinically managed by use of growth factors or by allogenic bone marrow transplantation (ABMT), whereas cardiotoxicity, hepatotoxicity could be circumvented by use of carnitine and its acetyl derivative known to have both cardioprotective and hepatoprotective effects.

Our hypothesis was to verify the beneficial role of ALC in modulating the adverse effects of mitoxantrone activity.

Over the past decades, numerous reports have shown the beneficial effect of carnitine and its acetyl-derivative in animals and humans.

The involvement of carnitine and its acetyl-derivative has been extensively reported. Biochemically, ALC acts as a carrier of fatty acids across the inner mitochondrial membrane for their subsequent beta-oxidation (12). It has been found that acetyl-L-carnitine hydrochloride increases the metabolic rate of mitochondria, improving the mitochondrial utilisation of oxygen $(4,24)$. ALC enhances the utilisation of alternative sources of energy (1).

Carnitine derivatives play a key role in regulating the flow of energetic substrates through cell membranes $(9,11,23)$. A decrease of fatty acid oxidation due to impairment of carnitine dependent mechanisms results in an impaired hepatic ketogenesis. When the carnitine dependent mechanisms are impaired, fatty acid oxidation is reduced and triglycerides accumulate, therefore resulting into both microscopic fatty change and impaired hepatic ketogenesis (13). 
Limited data are available concerning the mechanisms of tissue wasting and weight loss in cancer patients. Malnutrition associated with malignancy has been documented in many hospitalised cancer patients. This characteristic state of malnutrition and progressive tissue wasting is referred to as cancer cachexia and is believed to result from a combination of decreased nutrient intake, altered energy expenditure and abnormal substrate utilisation. However, recent literature suggests that this response is also a result of complex metabolic alterations and not only a result of starvation $(16,26)$. Thus the presence of cancer appears to cause metabolic alterations in the host $(22,26)$.

All these data suggest the probable beneficial role of nutritive (i.e. supportive) care in cancer treatment. When using ALC in combination with MX, we observed a substantial increase in length of survival of treated animals compared to MX alone. Intravenous application seems to be the best way of MX application. However intraperitoneal application was demonstrated as effective in this experiment. The contact with tumour cells in situ is probably necessary for the expression of the cytocidal effect of the drug.

Our hypothesis was not only to modulate the adverse effect of MX therapy but also to make a change in energetic balance in favour of the host. The clinical use of ALC as an adjuvans to MX and other antineoplastic agents may be a useful contribution in improving the metabolic state.

\section{Acknowledgements}

Anna Kargerová and Jitka Šedivá from the Research Institute for Pharmacy and Biochemistry Prague are acknowledged for technical assistance in experiments involving mice.

This work was supported by the Charles University grant no. 73/93 and the Research Institute of Organic synthesis of Pardubice (VÚOS a.s.).

\section{References}

1. Aureli T, Michelli R, Ricciolini M et al. Effect of acetyl-Lcarnitine treatment on rat brain energy and phospholipid metabolism. A study by ${ }^{31} \mathrm{P}$ and ${ }^{1} \mathrm{H}$ NMR spectroscopy. Brain Res 1989:526:108.

2. Bachur NR, Yu F. Johnson R. et al. Helicase inhibition by anthracycline anticancer agents. Mol Pharmacol 1992;41:993-8.

3. Beck WT, Danks MK, Wolverton JS et al. Resistance of mammalian tumour cells to inhibitors of DNA topoisomerase II. Adv Pharmacol 1994;29B:145-69.

4. Bellei MD m, Battelli DM, Guarriero U et al. Changes in mitochondrial activity caused by ammonium salts and the protective effect of carnitine. Biochim Biophys Res Commun 1989;158:181.
5. Benjamin RS. Rationale for the use of mitoxantrone in the older patient: cardiac toxicity. Semin Oncol 1995;22(Suppl.1):11-3.

6. Bezwoda WR, Bernasconi C, Hutshinton RM, Windfield DA, De Bock R, Mandelli F. Mitoxantrone for refractory and relapsed acute leukemia. Cancer 1990;96:418-22.

7. Carter WH Jr, Wampler GL, Stablein DM, Campbell ED. Drug activity and therapeutic synergism in cancer treatment. Cancer Res 1982;42:2963-71.

8. De Jager R, Capeleare P, Earl H et al. Phase II clinical trial of mitoxantrone on solid tumours and lymphomas. Trial NCI/EORTC. Symposium on New Drugs on Cancer Therapy. Brussels, Belgium: Institut Jules Bordet, 1998:Abst 39.

9. Delogu G, De Simone C, Famularo G, Fegiz A, Paoletti F, Jirilio E. Anaesthetics modulate tumour necrosis factor (: effect of L-carnitine supplementation in surgical patients. Preliminary results. Mediat Inflam 1993;2:33-6.

10. Endicott JA, Ling V. The biochemistry of p-glycoprotein mediated multidrug resistance. Annu Rev Biochem 1989;58:137-71.

11. Fattorossi A, Bisseli R, Casciaro A, Tzantzoglou S, De Simone C. Regulation of normal human polymorphonuclear leukocytes by carnitine. Mediat Inflam $1993 ; 2: 37-41$

12. Fritz IB. Carnitine and its role on fatty acid metabolism. Adv Lipid Res $1963 \cdot 1 \cdot 285-324$

13. Gasparetto A, Corbucci GG, De Blasi RA. Influence of acetyl-L-carnitine infusion on haemodynamic parameters and survival of circulatory - shock patients. Int J Clin Pharm Res XI 1991;2:83-92.

14. Hansson J. Mechanisms of tumour cell resistance to cancer chemotherapeutic drugs. Eur J Surg 1991;(Suppl 561):35-44.

15. Kenneth RH. Clinical applications of anticancer drugs targeted to topoisomerase II. Biochim Biophys Acta (BBA)/Gene Struct Expres 1998;1400:173-84.

16. Kern KA, Norton JA. Cancer cachexia. J Parent Ent Nutr 1988;12:286-92.

17. Koloničný A. Synthesis of Mitoxantrone (Ph.D.Thesis). Pardubice, Czech Republic: Technical University of Chemical Technology, 1990.

18. Minofti G. Adriamycin-dependent release of iron from microsomal membranes. Arch Biochem Biophys 1989;268:398-403.

19. Myers CE, McGuire WP. Liss RH. et al. Adriamycin: the role of lipid peroxidation in cardiac toxicity and tumour response. Science 1977;197:165-9.

20. Nielson D, Skovsgaard T. P-glycoprotein as multidrug transporters; a critical review of current multidrug resistant cell lines. Biochim Biophys Acta 1992;1139:169-83.

21. Pacciuci PA, Ohmuma T, Ambinder E. Effects of mitoxantrone on patients with refractory acute leukemia. Blood 1981;(Suppl 58):145.

22. Richards EW, Long CL, Nielson KM et al. Glucose metabolism in advanced lung cancer patients. Nutrition 1992;8:245-51.

23. Ruggiero V, D’Vrso M, Albertoni C, Campo S, Foresta P, Martelli EA. LPS - induced serum TNF production and lethality in mice: effect of L-carnitine and some acyl-derivatives. Mediat Inflam 1993:2:43-50.

24. Silliprandi ND, Lias F, Menabo R, Ciman M, Sartyoreli L. Transport functions of carnitine in muscles. J Clin Chem Clin Biochem 1990;28:303-6.

25. Tewey KM, Rowe TC, Yang L et al. Adriamycin-induced DNA damage mediated by mammalian DNA topoisomerase II. Science 1984;226:446-68.

26. Zadák Z, Shenkin A, Bláha V, Sobotka L, Cerman J, Solichová D. Metabolic response to glucose and insulin in weight-stable and weight loosing patients. Clin Biochem Metab 1996;25:125-30.

Submitted June 2000.

Accepted June 2000.

PharmDr. Mohamed Niang,

Charles University in Prague,

Faculty of Medicine in Hradec Králové, Department of Medical Biochemistry, Šimkova 870, 50001 Hradec Králové, Czech Republic. e-mail: niang@lfhk.cuni.cz 